\title{
The inflammatory accumulation of lipids and ROS in human Nrf1 $\alpha$-deficient hepatoma cells is ameliorated by 2-bromopalmitate
}

\author{
Ze Zheng ${ }^{1}$, Meng Wang ${ }^{1}$, Shaofan $\mathrm{Hu}^{1}$, Rongzhen Deng ${ }^{1}$, Jing Feng ${ }^{1}$, and Yiguo Khang $^{1}$
}

${ }^{1}$ The Laboratory of Cell Biochemistry and Topogenetic Regulation, College of Bioen gineering and Faculty of Medical Sciences,
Chongqing University, No. 174 Shazheng Street, Shapingba District, Chongqing 400044, China
* Correspondence: yiguozhang@cqu.edu.cn, or eaglezhang64@gmail.com

Abstract: Since Nrf1 and Nrf2 are essential for regulating the lipid metabolism pathways, their dysregulation was also shown to be critically involved in the non-controllable inflammatory pathology into cancer development. However, it is unknown that the interaction between Nrf1 and Nrf2 in the regulation of lipid metabolism, especially in hepatoma cells. Here, we have further explored the molecular mechanisms underlying their distinct regulation of lipid metabolism, by comparative analysis of changes in those lipid metabolism-related genes in $\mathrm{Nrfl}^{-/-}$and/or $\mathrm{Nrf2}^{--}$ cell lines relative to wild-type controls. The results revealed that loss of Nrf1 leads to disordered lipid metabolism; its lipid synthesis pathway was up-regulated by JNK-Nrf2-AP1 signaling, while its lipid decomposition pathway was down-regulated by the nuclear receptor PPAR-PGC1 signaling, resulting in severe accumulation of lipids as deposited in lipid droplets. By contrast, knockout of Nrf2 gave rise to decreases in lipid synthesis and uptake capacity. These demonstrate that Nrf1 and Nrf2 contribute to significant differences in the cellular lipid metabolism regulatory profiles and relevant pathological responses. Further experiments unraveled that lipid deposition in $\mathrm{Nrfl}^{-1-}$ cells was resulted from CD36 upregulation by activating the PI3K-AKT-mTOR pathway, leading to induction of the inflammatory response. Following treatment of $\mathrm{Nrf1}^{--}$cells with 2-bromopalmitate (2BP), it enabled the yield of lipid droplets to be strikingly alleviated, as companied by substantial abolishment of CD36 and critical inflammatory cytokines. Such $\mathrm{Nrf1}^{-/}$-led inflammatory accumulation of lipids and ROS was significantly ameliorated by 2BP. Overall, this study provides a potential strategy for cancer prevention and treatment by precision targeting of Nrf1, Nrf2, or both.

Key words: Nrf1; Nrf2; lipid metabolism; lipid droplets; JNK; AP1; mTOR; CD36; IL-6; ROS; 2-bromopalmitate

\section{Introduction}

Lipids as biological macromolecules are essential for energy storage and structural membranes, and also crucial for a variety of biological functions in most life processes, including cell growth, proliferation, differentiation, and movement. Therein, many types of lipids act as important biological signal molecules and thus exert diverse functionality in energy metabolism and cell homeostasis during the healthy life process. Contrarily, abnormal lipid metabolism can lead to a variety of physiological diseases. For instance, obesity caused by over-nutrition, nonalcoholic steatohepatitis (NASH), and type 2 diabetes are all related to the imbalance of lipid metabolism status (1). In addition to specific metabolic syndromes, the development of hepatocellular carcinoma (HCC) is also inseparable from lipid disorders (2). This is because most cancer cells were shown to have a higher rate of de novo lipid synthesis, leading to an increase in newly-synthesized fatty acids (e.g., glycerol phospholipids and cholesterol) that serve the raw materials so as to meet the needs for malignant growth and proliferation of cancer cells. Besides, the decomposing metabolism of lipids by $\beta$-oxidation in the mitochondria can also provide enough energy, when required for cancer cells (3).

In almost all living organisms, the lipids exist as a major stored form of triglycerides and cholesterol in lipid droplets (LDs), a kind of hydrophobic organelle with monolayer membrane structure that can mobilize fatty acids during specific life processes (4). Since the liver is the main metabolic organ of lipids in vertebrates, these lipids play a vital role in the liver's physiological homeostasis and pathological processes of many relevant diseases. Of note, liver-specific knockout of $\mathrm{Nrf1}$ [also called Nfe2/1, encoding a highly conserved membrane-bound transcription factor 
of NASH and malignant transformation into hepatoma (6). Such hepatocyte-specific loss of Nrf1 caused up-regulation of the cytochrome p450 family CYP4A and CYP1A1 genes, as fatty acid (FA) and cystine/cysteine contents increased, followed by severe steatosis, apoptosis, necrosis, and inflammation and fibrosis, eventually worsening and developing into liver cancer $(7,8)$. During this pathogenesis, significant activation of two lipid metabolism-related genes Lipin1 and PGC-16 was also shown in the murine liver-specific $\mathrm{Nrf1}^{-/-}$cells, implying that Nrf1 negatively regulates these two genes by its direct binding to the putative antioxidant response elements (AREs) located in their promoter regions (9).

Nrf2 (also encoded by Nfe2/1, as a water-soluble transcription factor of the CNC-bZIP family) has been proved to regulate lipid metabolism by mediating the expression of a number of lipid metabolism-related genes, such as those encoding ACLY, ACC1 and ELOVL6. It was found by Huang et al (10) that $\mathrm{Nrf}^{-/}$mice were manifested with a loss of their liver weight at six months of age, as accompanied by a marked decrease of the triacylglycerol contents in their livers. Such changes were shown to be attributable to $\mathrm{Nrf2}^{-/}$-leading up-regulation of lipogenic genes including PPARY, FASN, SCD1 and SREBP1, albeit they were proved to serve direct target genes of Nrf2 (10). The adipose-specific ablation of $\mathrm{Nrf2}$ caused a transient delay of high-fat diet-induced obesity by altering glucose, lipid and energy metabolism in the mice (11). Altogether, these demonstrate that Nrf2 acts as an important regulatory player in lipid metabolism. However, how Nrf1 and Nrf2 differentially govern (and inter-regulate) the lipid metabolism process, in particular liver cancer cells, is poorly understood to date.

In this study, we focus to determine the lipid synthesis and catabolism, as well as the mechanism related to lipid deposition, by conducting various experiments to examine yield of lipid droplets in $\mathrm{Nrfl}^{-1-}$ cells and relevant changes in its lipid metabolism gene expression as compared with those obtained from in $\mathrm{Nrf2}^{-1-}$ cells. The results unveiled that Nrf1 and Nrf2 have performed their respective regulatory functions in the lipid metabolism process of hepatoma cells through distinct molecular mechanisms. Further experiments also revealed that both factors are differentially involved in putative cytoprotection against the inflammatory responses to abnormal lipid metabolism caused by $\mathrm{Nrf1}^{--}$and/or $\mathrm{Nrf2}^{--}$. Interestingly, such lipid deposition and related inflammation responses are significantly reduced by a palmitic acid analogue, 2-bromopalmitate (2BP). Collectively, this study provides a novel potential strategy for the prevention and treatment of NASH and hepatoma by precision targeting of Nrf1, Nrf2, or both.

\section{Materials and Methods}

\subsection{Cell lines and culture}

The human hepatocellular carcinoma HepG2 cells (i.e., $\mathrm{Nrf1} / 2^{+/+}$) were obtained originally from the American Type Culture Collection (ATCC, Manassas, VA, USA). Additional two derived $\mathrm{Nrf1}^{-/}{ }^{-/}$and $\mathrm{Nrf2}^{-/}$cell lines were established in our laboratory by Qiu et al (12). The fidelity was confirmed by its authentication profiling and STR (short tandem repeat) typing map (by Shanghai Biowing Applied Biotechnology Co., Ltd). Before experimentation, they were allowed for growth in Dulbecco's modified Eagle's medium (DMEM), containing $25 \mathrm{mmol} / \mathrm{L}$ high glucose, $10 \%(\mathrm{v} / \mathrm{v})$ fetal bovine serum (FBS), 100 units $/ \mathrm{mL}$ penicillin-streptomycin, by being cultured in a $37^{\circ} \mathrm{C}$ incubator with $5 \% \mathrm{CO} 2$.

\subsection{Real-time quantitative $P C R$ analysis}

After all experimental cell lines reached $80 \%$ of their confluence, the total RNAs were firstly extracted with no treatment. In the parallel experiments, these cell lines were subjected to different treatments with specific inhibitors or siRNA (in order to interfere with cognate target gene expression). Subsequently, total RNAs were extracted by using an RNA extraction kit (TIANGEN, Beijing, China) and then reversely transcribed into single-stranded CDNA by a Reverse-Transcription Kit (Promega, USA). The mRNA expression level of each target gene in these cell lines was 
87 determined by real-time quantitative PCR (RT-qPCR) with each of the indicated pairs of their forward and reverse

88 primers (in Table 1).

89 Table 1. All oligonucleotides and antibodies used in this study.

90 (i) The primer pairs used for RT-qPCR analysis.

\begin{tabular}{lll}
\hline Gene Name & Forward Primers (5' to 3') & Reverse Primers (5' to 3') \\
\hline$\beta$-actin & GCGGCTACAGCTTCACCAC & GCCATCTCTTGCTCGAAGCCA \\
$N r f 2$ & CCCCTGCTCTTCAAAACAACC & ATCTTGCCACTGCTGTCC \\
$F A S N$ & AGTCCAGAAGCCAAACTGACA & ATGCTGCTGAAGGAATCCTCA \\
$S C D 1$ & ATTCGACCTTTCTCAGAACCAC & AAGAACGCATCCAGTAGGAC \\
ACC $\alpha$ & ATGCTAATCAATGCCGCAAC & CTTCCCCAGCAGAGACCAC \\
SREBP1 & CTCAGAGGCGAGAAACTCCA & CATCAATCGCTCTACCCAGT \\
$H O 1$ & GCCATCGACTACATTCGCTTT & GCACGTCTGTGTTCCCTCC \\
$J U N$ & CAGTGCCACCAAGTTCAACA & CTTGGCCTCTTCTATCACCCT \\
$N Q O 1$ & ATGGAAACGACCTTCTATGACGA & CGTTGCTGGACTGGATTATCA \\
$G C L M$ & ATTCCTTAGCCTGGATTTCCT & GCACGAATACAGTCGATTCCC \\
$G C L C$ & ACGAGAATCGCTTGAACCT & AGTTTTCCAGGCAAATCACA \\
$C D 36$ & CCCGATATGACTCAATAGACAGC & ATGATCAATGCCTTCCTGCAAC \\
$I L-6$ & TACAGATGCAGCCTCATTTCCAC & ACAGCATAGATTGACCTGCAAA \\
$I L-1 \beta$ & CAAATGTAGCATGGGCACCT & AACATAAGTTCTGTCCCAGT \\
$N F K B 1$ & CCTGCCCACAGACCTTCCAG & AAGCCTCGTTATCCCATGTGT \\
$N F \kappa B 2$ & CGTCAGAAGCTCATGCCCAA & GCCACTACCAAACATGCCTCC \\
$T N F \alpha$ & CCTGAAGCCAGTCATCTCCC & GCACCTTGTCACAAAGCAGA \\
\hline
\end{tabular}

(ii)

Oligonucleotides sequences of siRNA.

\begin{tabular}{ccc}
\hline Name & Forward Primers (5' to 3') & Reverse Primers (5' to 3') \\
\hline siNrf2 & GUAAGAAGCCAGAUGUUAAdTdT & UUAACAUCUGGCUUCUUACdTdT \\
siJUN & GCAUGGACCUAACAUUCGAdTdT & UCGAAUGUUAGGUCCAUGCdTdT \\
\hline
\end{tabular}

92

(iii) The key antibodies used in this work

\begin{tabular}{|c|c|c|}
\hline Antibodies & Identifier & Source \\
\hline Nrf1 & N/A & Zhang's(13) \\
\hline Nrf2 & ab62352 & Abcam \\
\hline $\mathrm{ACC} \alpha$ & D155300 & Sangon Biotech \\
\hline FASN & D262701 & Sangon Biotech \\
\hline SCD1 & D162163 & Sangon Biotech \\
\hline SREBP1 & 14088-1-AP & Proteintech \\
\hline ATGL & ab109251 & Abcam \\
\hline $\operatorname{PPAR} \alpha$ & D161086 & Sangon Biotech \\
\hline $\operatorname{PPAR} \gamma$ & ab272718 & Abcam \\
\hline $\operatorname{PPAR} \dot{\delta}$ & ab178866 & Abcam \\
\hline PGC-1 $\alpha$ & D262041 & Sangon Biotech \\
\hline PGC- $1 \beta$ & D162042 & Sangon Biotech \\
\hline JNK & ab208035 & Abcam \\
\hline p-JNK & ab124956 & Abcam \\
\hline JUN & 10024-2-AP & Proteintech \\
\hline FOS & ab134122 & Abcam \\
\hline $\mathrm{HO} 1$ & ab68477 & Abcam \\
\hline AKT1 & ab32505 & Abcam \\
\hline $\mathrm{p}-\mathrm{AKT}$ & ab38449 & Abcam \\
\hline p-mTOR ${ }^{\angle 440}$ & ab109268 & Abcam \\
\hline p-mTOR ${ }^{2481}$ & ab137133 & Abcam \\
\hline $\mathrm{PI} 3 \mathrm{~K}$ & $a b 245781$ & Abcam \\
\hline CD36 & ab133625 & Abcam \\
\hline $\mathrm{COX} 2$ & ab62331 & Abcam \\
\hline IL-6 & $a b 233706$ & Abcam \\
\hline $\mathrm{NF \kappa B}$ & ab32360 & Abcam \\
\hline p-NFкB & ab140751 & Abcam \\
\hline$\beta$-actin & TA-09 & ZSGB-BIO \\
\hline
\end{tabular}




\subsection{Small interfering RNAs and kinase-specific inhibitors}

To determine the effect of a given gene on the downstream signaling, the JNK function inhibitor SP600125 (Selleck, Shanghai, China), the PI3K inhibitor NVP-BKM120 (Cayman Chemical, USA), and the mTOR inhibitor RAPA (rapamycin, Sigma, USA) were employed respectively. Two pairs of small-interfering RNAs (i.e., siRNAs, with their oligonucleotide sequences listed in Table 1) were designed for targeting Nrf2 and JUN that interfere with their mRNA expression levels, before being synthesized by Tsingke (Beijing, China).

\subsection{Western blotting analysis of key proteins}

All experimental cells were subjected to extraction of total proteins in a lysis buffer, containing a protease inhibitor cOmplete Tablets EASYpack or another phosphatase inhibitor PhosSTOP EASYpack (each tablet per $15 \mathrm{~mL}$ buffer, Roche, Basel, Switzerland). The proteins in cell lysates were denatured by boiling at $100^{\circ} \mathrm{C}$ for 10 min, disrupted by ultrasound, and then diluted in $3 \times$ loading buffer $(187.5 \mathrm{mmol} / \mathrm{L}$ Tris- $\mathrm{HCl}, \mathrm{pH} 6.8,6 \% \mathrm{SDS}, 30 \% \mathrm{Glycerol}, 150 \mathrm{mmol} / \mathrm{L}$ DTT, and $0.3 \%$ Bromphenol Blue) at $100^{\circ} \mathrm{C}$ for $5 \mathrm{~min}$. Thereafter, equal amounts of proteins were separated by SDS-PAGE gels containing $8-12 \%$ polyacrylamide and visualized by Western blotting with distinct primary antibodies (listed in Table 1).

\subsection{Determination of intracellular triglyceride contents}

All the relevant experimental cells were subjected to determination of the triglyceride (TG) contents, as conducted according to the manufacture's instruction of the TG-enzymatic assay kit (APPLYGEN, Beijing, China).

\subsection{Oil Red O-staining of lipid droplets in cells}

After all experimental cells reached $70 \%$ of their confluence, the cells were subjected to different treatments with either different concentrations of oleic acid (OA, Solarbio, Beijing, China) or 2-bromopalmitate (2BP, Sigma, USA). Then, the cells were fixed in a tissue fixative buffer containing 4\% paraformaldehyde (Boster Biological Technology, Wuhan, China) for $30 \mathrm{~min}$, prior to being stained for additional $30 \mathrm{~min}$ in another solution containing $3 \mathrm{~g} / \mathrm{L}$ of oil red O (Sangon Biotech, Shanghai, China). Subsequently, the stained cells were rinsed three times by using $60 \%$ of isopropyl alcohol (Kelong, Chengdu, China), before the red lipid droplets were visualized by microscopy and photographed as shown.

\subsection{Analysis of cell ROS by flow cytometry}

After all experimental cells reached $70 \%$ of their confluence, the cells were transferred to be cultured in a medium containing 2BP or without this chemical for $24 \mathrm{~h}$ and then prepared according to the manufacture's instruction of the ROS assay kit (Beyotime, Shanghai, China). Thereafter, the intracellular ROS levels were determined by flow cytometry. The FlowJo 7.6.1 software, and the resulting data were analyzed as described previously (14).

\subsection{Statistical analysis}

Significant differences were statistically determined using the Student's t-test and Multiple Analysis of Variations (MANOVA), except for somewhere indicated. The data are herein shown as a fold change (mean \pm S.D.), each of which represents at least three independent experiments that were each performed in triplicate.

\section{Results}

\subsection{Knockout of Nrf1 $\alpha$ leads to lipid deposition while knockout of Nrf2 does the opposite in hepatoma cells.}

A striking feature in the pathology of NASH is lipid deposition, which is also a hallmark of liver cancer development from the murine $\mathrm{Nrf1}^{-/-}$-disordered lipid metabolism $(7,8)$. Herein, wild-type HepG2 cells $\left(\mathrm{Nrf1} / 2^{+/+}\right.$) and two derived cell lines ( $\mathrm{Nrf}_{1} \alpha^{-/}$and $\mathrm{Nrf2}^{-/}$) were employed in the following experiments to investigate distinct functionality of Nrf1 and Nrf2 in human intracellular lipid metabolism. These cell lines were further validated by Western blotting and real-time qPCR analyses of Nrf1 and Nrf2 (Fig. 1, A \& B); the results were consistent with our 
134 previous data $(12,14)$. When compared with wild-type $\mathrm{Nrf1} / 2^{+/+}$controls, $\mathrm{Nrf1} \alpha^{-/-}$cells exhibited no expression of

$135 \mathrm{Nrf1} \alpha$-derived proteins, but other shorter isoforms of $\mathrm{Nrf1ß}$ and $\mathrm{Nrf1} \gamma$ were almost unaltered (Fig.1 A, upper panel).

136 By contrast, Nrf2 protein abundance (Fig.1 A, middle panel) and its basal mRNA levels (Fig. 2B) were significantly

137 increased in $\mathrm{Nrf}_{1} \alpha^{--}$cells. Conversely, Nrf1 $\alpha$-derived proteins were slightly enhanced in $\mathrm{Nrf}^{-/}$cells (lacking its two 138 major proteins), but this was accompanied by a modest decrease in basal mRNA expression of Nrf1 (Fig. 1, A \& B).

139 These indicate putative inter-regulation of between $\mathrm{Nrf} 1 \alpha$ and $\mathrm{Nrf} 2$ could be executed at distinct layers.

140 After 24-h treatment of distinct cell lines with oleic acid (OA) at different concentrations $(0,50,100 \mu \mathrm{mol} / \mathrm{L})$, the 141 yields of lipid droplets were evaluated by oil red $O$ staining. The resulting images were photographed and shown in 142 (Fig. 1D). When compared with wild-type $\mathrm{Nrf1} / 2^{+/+}$controls, much more and larger lipid droplets were yielded in $143 \mathrm{Nrf}_{1} \alpha^{--}$cells, whereas the least and smallest lipid droplets were in $\mathrm{Nrf2}^{--}$cells. Also, yields of lipid droplets after OA 144 treatment of the above three cell lines were increasing to different extents, with incrementing concentrations of OA 145 from 50 to $100 \mu \mathrm{mol} / \mathrm{L}$ (Fig. 1D). Such an OA-increased trend of lipid droplets in $\mathrm{Nrf1}^{-/}$cells was significantly higher 146 than that of $\mathrm{Nrf}_{1 / 2^{+/+}}$controls, while the lowest trend of increased lipid droplets was manifested in $\mathrm{Nrf2}^{-/-}$cells. 147 These unraveled that loss of $\mathrm{Nrf1} \alpha$ led to an aberrant accumulation of lipids, whereas similar lipid deposition 148 appeared to be alleviated by Nrf2 inactivation. This notion was further evidenced by an intracellular triglyceride (TG) 149 enzymatic detection assay of the above experimental cell lines that had been treated with $100 \mu \mathrm{mol} / \mathrm{L} \mathrm{OA} \mathrm{(Fig.} \mathrm{1C).}$ 150 Their distinct TG contents (in particular lipid droplets) were also visualized by oil red $O$ staining, as shown 151 photographically in (Fig. 1E). These distinct results demonstrated that loss of Nrf1 $\alpha$ enables the lipid deposition to be 152 augmented in $\mathrm{Nrf1}^{-/-}$hepatoma cells, but a similar lipid-accumulating phenomenon was not observed in $\mathrm{Nrf2}^{--}$cells 153 and even its lipid synthesis and/or uptake may be also suppressed by Nrf2-specific knockout of its transactivation 154 domain rather than its DNA-binding domain.

\subsection{Loss of Nrf1 $\alpha$ or Nrf2 causes different effects on the expression of genes responsible for lipid metabolism.}

It had been shown that those genes governing the de novo lipid synthesis were highly expressed in cancer cells to considerably greater extents than their equivalents in normal cells (15). Such key rate-limiting enzymes (e.g., FASN, $A C C \alpha, S C D 1$ ) of de novo lipid synthesis and regulated transcriptional factor SREBP1 (sterol regulatory element-binding protein 1) were examined herein. As anticipated, the results revealed that loss of $\mathrm{Nrf1}^{-/-}$caused substantial increases in the protein expression of such key genes encoding FASN (fatty acid synthase), ACC $\alpha$ (acetyl-COA carboxylase alpha), SCD1 (stearoyl-Coenzyme A desaturase 1) and SREBP1 (Fig. 2A), when compared with wild-type $\mathrm{Nrf1} / 2^{+/+}$cells. Similar augmented expression of their mRNA levels in $\mathrm{Nrf1}^{-/-}$cells were also determined by real-time qPCR (Fig. 2B). By sharp contrast, Nrf2 ${ }^{-/}$cells gave rise to down-expressed levels of these genes to greater or fewer degrees (Fig. 2, A \& B).

Further examinations unraveled a considerably lower expression level of adipose triglyceride lipase (ATGL, also called PNPLA2, which is the catalytic rate-limiting enzyme in the first step of TG hydrolysis during oxidation catabolic mobilization (16)) in $\mathrm{Nrf1}^{-1-}$ cells (Fig. 2, C \& D). Intriguingly, another modestly decreased expression of ATGL was also exhibited in $\mathrm{Nrf}^{--}$cells as compared with wild-type cells. Hence, to clarify this issue, further experimental analysis of those nuclear receptor-types (i.e. PPAR $\alpha, \gamma, \delta$ ) transcription factors governing oxidative degradation of fatty acids (17) and their interacting transcriptional co-activators (i.e. PGC-1 $\alpha, \beta)(18)$ was conducted herein. The results unraveled that all the examined proliferator activated receptors (PPAR $\alpha, \gamma, \delta$ ) and PPAR $\gamma$ co-activators (PGC-1 $\alpha$, -1ß) were significantly down-regulated in $\mathrm{Nrf1}^{-/}$cells (retaining a hyper-active Nrf2), and thus were roughly unaffected in $\mathrm{Nrf2}^{--}$cells but with an exception of downexpressed PPAR $\alpha$ only (Fig. 2, C \& D). Taken altogether, these collective data demonstrate that aberrant deposition of lipids in $\mathrm{Nrfl}^{-\alpha^{--}}$cells result from up-regulation of lipid anabolism, along with substantial down-expression of lipid catabolism-controlled genes, but their opposite effects were elicited in $\mathrm{Nrf2}^{--}$cells. 
Such differential expression profiles of lipid synthesis- and catabolism-related genes in between $N r f 1 \alpha-$ and Nrf2-deficient cell lines were further explored. The results showed a markedly increased abundance of phosphorylated JNK and its cognate target JUN in $\mathrm{Nrf1}^{-/}$cells (with a hyper-active Nrf2) (Fig. 2E, e2 to e4), although the total protein level of JUK was relatively down-expressed (Fig. 2E, e1), when compared with wild-type controls. By sharp contrast, all those examined protein levels were strikingly diminished upon inactivation of $\mathrm{Nrf2}^{-/-}$(Fig. 2E). Such similar expression trends of Nrf2, p-JUNK and JUN suggest a comparable pattern to those of the aforementioned lipogenic genes, thus providing a new clue for the further study of their relevant gene regulation.

\subsection{The JNK-Nrf2-AP1 signaling is required for up-regulation of lipogenic genes in Nrf1 - -deficient cells.}

Here, treatment of $\mathrm{Nrf}_{1} \alpha^{-/-}$cells with a specific inhibitor SP600125 (to block the activity of phosphorylated JNK, as shown in Fig. 3A, a1) caused substantial down-expression of all seven other examined proteins, including Nrf2, JUN (a direct substrate of JNK), HO-1, FASN, ACC $\alpha$, SCD1 and SREBP1 (Fig. 3A, a2 to a8). Of note, the lipid synthesis-related $A C C \alpha$ and SCD1 displayed a profound inhibitory trend similar to those of JUN and Nrf2, albeit SREBP1 was only partially inhibited by SP600125. This implies that the JUK signaling to Nrf2 and/or AP1 is involved in the aberrant lipogenesis of $\mathrm{Nrf1}^{-/-}$cells. As such, the treated cells still retained a remnant fraction of JUN and Nrf2, but their direct downstream HO-1 expression was almost completely abolished, implicating an integrative effect of both factors on $\mathrm{HO}-1$.

Further experiments revealed that silencing of Nrf2 by its siNrf2 (to interfere with its expression in $\mathrm{Nrf1}^{-/-}$cells) also led to obvious down-regulation of AP1 (i.e. a heterodimer of JUN and FOS), HO-1 (a direct target of Nrf2 and AP1), and those lipogenic enzymes FASN, ACC $\alpha$ and SCD1 (Fig. 3B, b1 to b6). Next, the real-time qPCR analysis unraveled that knockdown of Nrf2 by its siNrf2 caused significantly reduced expression of JUN, FASN, ACC $\alpha$ and SCD1 (Fig. 3C). This indicates a predominant requirement for Nrf2 in regulating AP1 and the key enzymes in lipid synthesis, besides its cognate target genes HO-1, NQO1, GCLC and GCLM (Fig. 3C). However, SREBP1 appeared to be roughly unaffected by silencing of Nrf2 (Fig. 3, B \& C).

Additionally marked decreases in the expression of FASN, ACC $\alpha$ and SCD1 at their protein and mRNA levels resulted from siJUN-led knockdown of AP1 activity in $\mathrm{Nrf1} \alpha^{-/-}$cells (Fig. 3, D \& E). Nonetheless, SREBP1 was almost unaltered by silencing of JUN. Further examination of $\mathrm{Nrfl}^{-/}$cells by transfecting expression constructs for JUN alone or plus FOS revealed that such forced expression of AP1 led to striking up-regulation of SCD1, but FASN was unaffected (Fig. 3F, c.f. f5 with f3). Intriguingly, only a marginal increase of ACC $\alpha$ was caused by overexpression of JUN alone, but conversely, a substantial decrease of this enzyme was dictated by co-expression of JUN and FOS (Fig. 3F, f4). Such dual opposite regulation of $A C C \alpha$ by JUN alone or plus FOS may be determined by a putative functional heterodimer of JUN with another partner, competitively together with the AP1 factor, depending on distinct experimental settings.

\section{4 $\mathrm{Nrf1}^{-/-}$cell-autonomous induction of the PI3K-AKT-mTOR signaling leads to abnormal hyper-expression of CD36.}

An important nutritional metabolism mechanism sets into account by signaling of the PI3K (phosphatidylinositol 3-kinase) to its downstream AKT (also called protein kinase B) and mTOR (mechanistic target of rapamycin kinase) (19). Upon activation of mTOR by stimulation of nutritional signals, it enables for up-regulation of CD36 (acting as a regulator of fatty acid uptake and its transport (20). As anticipated, a significant increase in basal constitutive expression of CD36 was determined in $\mathrm{Nrf1}^{-/}$cells, when compared with wild-type controls (Fig. 4A, a4). Such aberrant hyper-expression of $\mathrm{CD} 36$ was accompanied by dominant cell-autonomous activation of $\mathrm{PI} 3 \mathrm{~K}^{\mathrm{P} 110} \triangle$ and $\mathrm{AKT}$, as well as of phosphorylated AKT and mTOR at distinct sites (Fig. 4A, a1 to a5). In addition, Nrf1 $\alpha^{-/-}$cells gave rise to the basally-augmented expression of a general inflammatory marker COX2 (cyclooxygenase 2) (Fig. (Fig. 4A, a7); this enzyme was also called prostaglandin-G/H synthase (PTGS2), involved in the arachidonic acid metabolism. 
In order to determine whether $\mathrm{Nrf}_{1} \alpha^{-/-}$cell-autonomous activation of the PI3K-AKT-mTOR signaling is required for up-regulation of CD36 by its deficiency, a PI3K-specific inhibitor NVP-BKM120 (Fig. 4B) and another mTOR-specific inhibitor rapamycin (RAPA, Fig. 4C) were allowed for treatment of $\mathrm{Nrf} 1 \alpha^{-/}$and $\mathrm{Nrf1/2^{+/+ }}$ cell lines respectively. As expected, the results revealed that two phosphorylated protein levels of mTOR were substantially diminished by NVP-BKM120 and RAPA in their treated cell lines (Figs. 4B, b1 \& b2 and 4C, $c 1 \& c 2$ ). In the meantime, basal expression abundances of COX 2 and CD36 were also almost abrogated by NVP-BKM120 and RAPA (Figs. 4B, $b 7 \& b 8$ and $4 C, c 8 \& c 9)$. However, no or less effects of both inhibitors on all other examined proteins (e.g., JNK, Nrf1, Nrf2 and $\mathrm{HO}-1$ ) were detected (Figs. $4 \mathrm{~B}, b 3$ to $b 6$ and $4 \mathrm{C}, c 3$ to $c 7$ ). Altogether, these demonstrate that loss of Nrf1 $\alpha$ causes a non-controllable activation status of CD36, as well as COX2, primarily by the PI3K-AKT-mTOR signaling pathway.

Such up-regulation of CD36 can also enable it to assist the Toll-like receptor 4 (TLR4) with synergistic activation of the inflammatory factor NFKB and cytokines IL-6, IL-1 $\beta$ and TNF $\alpha$ (21). Next, we thus determine whether their changed expression levels in $\mathrm{Nrf1}^{-/ \alpha^{-}}$cells have relevance to the hyper-expression of CD36. The results unveiled that dramatic activation of $C D 36$ by loss of Nrf1 $\alpha$ was accompanied by varying enhancements of NFKB, IL-6, IL-1B, and TNF $\alpha$ at their protein and/or mRNA expression levels in $\mathrm{Nrfl}^{-/}$cells, when compared with those equivalents of $\mathrm{Nrf1} 1 / 2^{+/+}$cells (Fig. 4, D \& E). Overall, it is postulated that $N r f 1 \alpha^{-/-}$-disordered lipid metabolism may be attributable to aberrant activation of $\mathrm{CD} 36$, with a concomitant inflammatory response.

\subsection{The inflammatory accumulation of lipids and ROS in $\mathrm{Nrfl}^{-1 /-}$ cells is alleviated by 2-bromopalmitate.}

As shown schematically in Fig. $5 \mathrm{~A}, 2 \mathrm{BP}$ is a palmitic acid analogue with one bromine $(\mathrm{Br})$ atom at the $\beta-C$ position, allowing for dramatic improvement of its stability more than that of the prototypic palmitate so as to make it difficult to proceed to the next step. Thereby, this physicochemical property of 2BP can enable this palmitic acid analogue to block those biological processes in which palmitic acids should have participated; for example, 2BP was employed as a natural ligand of PPARS receptor in fatty acid oxidation (22). Herein, it was found that the triglyceride (TG) accumulation in $\mathrm{Nrf1} \alpha^{-/}$cells was significantly inhibited by 2BP (Fig. 5B). Similar treatments with 2BP caused almost abolishment of basal CD36 expression in both $\mathrm{Nrf1}^{-/-}$and $\mathrm{Nrf1} / \mathrm{2}^{+/+}$cell lines (Fig. $5 \mathrm{C}$ ), and also enabled for substantial suppression of lipid droplets in $\mathrm{Nrf1}^{-/-}$cells to considerably lower levels (Fig. 5D). These findings demonstrate that 2-bromopalmitate has the capability to alleviate severe lipid deposition mediated by CD36 in Nrf1 $1 \alpha^{-1-}$ cells.

Further examination revealed that transcriptional expression levels of the inflammatory cytokines IL-6 and TNF $\alpha$ in $\mathrm{Nrf1}^{-/}$cells were significantly suppressed or even completely abolished by 2BP, respectively, when compa red with wild-type controls (Fig. 5E). Also, the basal IL-6 abundance and active phosphorylated NFKB were apparently repressed by $2 \mathrm{BP}$ (Fig. $5 \mathrm{C}$ ). This implies that the informatory response to the loss of $\mathrm{Nrf1}^{-/-}$may be ameliorated by 2-bromopalmitate. Flow cytometry analysis unraveled a marked accumulation of the intracellular reactive oxygen species (ROS, that could be involved in the informatory response) in $\mathrm{Nrfl}^{-\alpha^{-}}$cells (Fig. 5F, left panel, with an enhanced peak shifted to the right), but such severe ROS accumulation was also obviously mitigated by 2BP (Fig. 5F, right panel, with a reduced peak shifted to the left). However, no similar ROS changes were observed in $\mathrm{Nrf1/2^{+/+ }}$ cells (middle panel). Collectively, these indicate that 2-bromopalmitate can enable effective alleviation of the inflammatory accumulation of lipids by enhanced biosynthesis and uptake of fatty acids (through up-regulation of CD36) in $\mathrm{Nrf1}^{-/-}$cells. This occurs concomitantly with a reduction of ROS by 2-bromopalmitate to mitigate the inflammatory response caused by loss of $N r f 1 \alpha$.

\section{Discussion}

In the present study, we have provided the evidence unraveling different or even opposite contributions of Nrf1 and Nrf2 have been made in human lipid metabolism, in addition to our previously-reported glucose metabolism 
$(14,23)$, protein homeostasis (proteostasis) $(12,24)$, redox balance and signaling control $(25,26)$. It is of crucial significance to note that the experimental cell model of $\mathrm{Nrfl}^{-1-}$ with pathophysiological phenotypes appears to largely resemble those manifested by the liver-specific Nrf1 knockout mice. Such loss of $\mathrm{Nrf1}^{- \text {- }}$ results in the spontaneous development of NASH and eventual liver cancer, which is attributed to the rapid accumulation of a large amount of lipids, as a key etiology, in the pathological process (6). Here, our experimental evidence demonstrated that various piles of lipids (i.e., triglyceride contents) were markedly deposited in distinct sizes of the intracellular lipid droplets widely distributed within human $\mathrm{Nrfl}^{-\alpha^{-1}}$ hepatoma cells, particularly following treatment with oleic and palmitic acids. By contrast, $\mathrm{Nrf2}^{--}$cells seemed to be considered destitute of lipid droplets, even after they were treated with oleic acids.

Interestingly, we have also discovered that aberrant lipid deposition in $\mathrm{Nrf1}^{-1-}$ cells was substantially prevented by a palmitic acid analogue, i.e., 2-bromopalmitate, through significant down-regulation of CD36 (acting as an essential translocator of fatty acids). The concomitant inflammatory response and oxidative stress were ameliorated significantly by 2-bromopalmitate. As a preventive consequence, key inflammatory cytokines and relevant transcription factor NFKB were markedly down-regulated, following apparent reduction of ROS and lipids in 2-bromopalmitate-treated $\mathrm{Nrfl}^{-1-}$ cells (as illustrated in Fig. 5). However, it should also be noted that, in addition to the secondary yield of ROS stimulated by the accumulation of lipids, the primary amount of ROS is produced by $\mathrm{Nrf} 1 \alpha^{-/}$cell-autonomous reprogramming of redox metabolism $(14,23,26)$. Thereby, such $\mathrm{Nrf1}^{-\alpha^{-}}$cell-autonomous yield of ROS is inferable to be almost unaffected by 2-bromopalmitate. In fact, our evidence has been presented herein, showing that even though the putative concomitant ROS resulting from severe lipid deposition may be diminished by this palmitic acid analogue, the entire oxidative stress is not completely mitigated and recovered to the levels measured from wild-type cells.

We have also provided evidence revealing that aberrant accumulation of lipids in $\mathrm{Nrf1} \alpha^{-/}$cells result from incremented uptake of fatty acids through constitutive activation of the PI3K-AKT-mTOR signaling to up-regulation of CD36 (as shown in Fig. 5). The CD36-augmented uptake of lipids is fully consistent with those results obtained from the tissue-specific $\mathrm{Nrf1}^{-/}$mice $(7,27)$. These two independent groups also unraveled that Nrf1 is essential for controlling translocation of fatty acids and cystine/cysteine contents by governing a complex hierarchical regulatory network in the redox and lipid metabolism. Therein, some cytochrome family members, besides CD36, were also up-regulated by loss of $\mathrm{Nrf1}^{--}$, thus leading to a certain yield of ROS from the oxidative metabolism of fatty acids, in the murine deficient liver cells $(7,8)$. In addition, it is also quite interesting to notice our previous work unraveling that Nrf1 and Nrf2 are contributable to dual opposing regulation of the PTEN-AKT signaling to be selectively repressed or activated, respectively, in the pathogenesis of NASH and liver cancer (12). Here, we further validated that the extended PI3K-AKT-mTOR signaling to the inflammatory marker COX, as well as those key inflammatory responsive factors along with CD36, was also constitutively activated by loss of Nrf1 $\alpha$ in human hepatoma cells.

In this study, we have further provided a revealing insight into the molecular mechanisms of $\mathrm{Nrf1}^{-1}{ }^{- \text {-leading }}$ lipid metabolism disorders, along with a cascade of the enzymatic reactions leading to lipid deposition during the development of NASH and hepatoma. The resulting evidence demonstrates that those key enzymes including FASN, SCD1 and ACC $\alpha$ and relevant transcriptional factor SREBP1 involved in the de novo biosynthesis of lipids were significantly up-regulated in $\mathrm{Nrf1}^{-/}$cells with hyper-active $\mathrm{Nrf2}$, but they were hence unaffected or even down-regulated by inactivation of $\mathrm{Nrf} 2$ in $\mathrm{Nrf}^{-1-}$ cells. Further experimental evidence unraveled that such up-regulation of the lipid biosynthesis pathway by $\mathrm{Nrf1}^{-\alpha^{-}}$-deficiency may occur through the JNK-Nrf2-AP1 signaling. Of note, a few of different experimental results may be determined by distinct functional heterodimers of Nrf2 with each of sMaf or other bZIP proteins, thus competitively with JUN and FOS against the heterodimeric AP1 factor for selective physical binding to the consensus ARE sites within their target gene promoter regions (28).

Furthermore, we also found that ATGL and the nuclear receptor PPAR-PGC1 signaling towards the lipid oxidative 
decomposition pathway were markedly down-regulated in $\mathrm{Nrf} 1 \alpha^{--}$cells, but they were unaffected or even conversely up-regulated in $\mathrm{Nrf2}^{-1-}$ cells. Such activation of the PPAR-PGC1 signaling was also supported by the evidence obtained from the tissue-specific $\mathrm{Nrf1}^{-/-}$mice $(9,29)$. Therein, Hirotsu et al $(9)$ had shown that liver-specific Nrf1 deficiency cannot only lead to obviously decreased expression of Lipin1 and PGC-1 $\beta$, but conversely, the presence of Nrf1 enabled this CNC-bZIP factor to directly mediate transcriptional activation of Lipin 1 and PGC-1 $\beta$ by binding to the ARE sequences in their promoter regions. Collectively, these indicate that Nrf1 is essential for regulating critical genes involved in lipid catabolism. Upon loss of $\mathrm{Nrf} 1 \alpha^{--}$, constitutive activation of the PPAR-PGC1 signaling is implicated in the severe aberrant accumulation of lipids. The ensuing metabolic pathological process may be prevented by 2-bromopalmitate, because this chemical had also been shown to serve as a natural ligand of PPARס receptor involved in the fatty acid oxidation (22), albeit detailed molecular mechanisms remain elusive.

In summary, this work demonstrates that both Nrf1 and Nrf2 make different or even opposite contributions to the intracellular lipid metabolism, as shown in the model of Fig. 6. Upon knockout of $\mathrm{Nrf1} \alpha$, the de novo biosynthesis of lipids is incremented by significant induction of the JNK-Nrf2-AP1 signaling pathway in $\mathrm{Nrf1}^{-/}$cells, while the lipid oxidative decomposition pathway was down-regulated by the PPAR-PGC1 signaling. Nrf1 $\alpha^{-/}$cell-autonomous activation of PI3K-AKT-mTOR signaling towards up-regulation of CD36 leads to augmented uptake of fatty acids. These together result in severe accumulation of lipids as deposited in lipid droplets. By contrast, $\mathrm{Nrf2}^{--}$cells gave rise to obvious decreases in both lipid synthesis and uptake capacity, along with enhanced oxidative metabolism of fatty acids. Importantly, such $\mathrm{Nrf}_{1 \alpha^{-1-}}$-leading lipid metabolism disorders and relevant inflammatory responses are substantially ameliorated by 2-bromopalmitate. Overall, this study provides a potential strategy for prevention of the inflammatory transformation into cancer and relevant treatment by precision targeting of Nrf1, Nrf2, or both in translational medicine.

Author contributions: Z.Z. performed the experiments with help of M.W., S.H., and J.F., collected all the relevant data, made draft of this manuscript with most figures and supplemental information with assistance of. R.D. Lastly, Y.Z. designed and supervised this study, analyzed all the data, helped to prepare all figures with cartoons, wrote and revised the paper.

Acknowledgments: We are greatly thankful to Drs. Lu Qiu (Zhengzhou University, Henan, China) and Yonggang Ren (North Sichuan Medical College, Sichuan, China) for their involvement in establishing the indicated cell lines used in this study. We also thank to all those other present and past members of Prof. Zhang's laboratory (at Chongqing University, China) for giving critical discussion and invaluable help with this work. Notably, this study was also funded by the National Natural Science Foundation of China (NSFC, with two project grants 81872336 and 82073079 ) awarded to Prof. Yiguo Zhang.

Conflicts of Interest: The authors declare no conflict of interest. Besides, it should be noted that the preprinted version of this paper had been initially posted at BioRxiv, doi: https://doi.org/10.1101xxx

\section{References}

1. Cheng, C., Zhuo, S. M., Zhang, B., Zhao, X., Liu, Y., Liao, C. L., Quan, J., Li, Z. Z., Bode, A. M., Cao, Y., and Luo, X. J. (2019) Treatment implications of natural compounds targeting lipid metabolism in nonalcoholic fatty liver disease, obesity and cancer. Int J Biol Sci 15, 1654-1663

345 4. Walther, T. C., and Farese, R. V., Jr. (2012) Lipid droplets and cellular lipid metabolism. Annu Rev Biochem 81, 687-714

5. Zhang, Y., and Xiang, Y. (2016) Molecular and cellular basis for the unique functioning of Nrf1, an indispensable transcription

2. Alannan, M., Fayyad-Kazan, H., Trezeguet, V., and Merched, A. (2020) Targeting Lipid Metabolism in Liver Cancer. Biochemistry-Us 59, 3951-3964

3. Santos, C. R., and Schulze, A. (2012) Lipid metabolism in cancer. Febs J 279, 2610-2623 factor for maintaining cell homoeostasis and organ integrity. Biochem J 473, 961-1000

6. Parola, M., and Novo, E. (2005) Nrf1 gene expression in the liver: A single gene linking oxidative stress to NAFLD, NASH and hepatic tumours. J Hepato/ 43, 1096-1097

7. Tsujita, T., Peirce, V., Baird, L., Matsuyama, Y., Takaku, M., Walsh, S. V., Griffin, J. L., Uruno, A., Yamamoto, M., and Hayes, J. D. 
(2014) Transcription Factor Nrf1 Negatively Regulates the Cystine/Glutamate Transporter and Lipid-Metabolizing Enzymes. Mol Cell Biol 34, 3800-3816

8. Xu, Z. R., Chen, L. Y., Leung, L., Yen, T. S. B., Lee, C., and Chan, J. Y. (2005) Liver-specific inactivation of the Nrf1 gene in adult mouse leads to nonalcoholic steatohepatitis and hepatic neoplasia. P Nat/ Acad Sci USA 102, 4120-4125

9. Hirotsu, Y., Hataya, N., Katsuoka, F., and Yamamoto, M. (2012) NF-E2-Related Factor 1 (Nrf1) Serves as a Novel Regulator of Hepatic Lipid Metabolism through Regulation of the Lipin1 and PGC-1 beta Genes. Mol Cell Bio/ 32, 2760-2770

10. Huang, J. S., Tabbi-Anneni, I., Gunda, V., and Wang, L. (2010) Transcription factor Nrf2 regulates SHP and lipogenic gene expression in hepatic lipid metabolism. Am J Physiol-Gastr L 299, G1211-G1221

11. Zhang, L., Dasuri, K., Fernandez-Kim, S. O., Bruce-Keller, A. J., and Keller, J. N. (2016) Adipose-specific ablation of Nrf2 transiently delayed high-fat diet-induced obesity by altering glucose, lipid and energy metabolism of male mice. Am J Trans/ Res 8, 5309-+

12. Qiu, L., Wang, M., Hu, S. F., Ru, X. F., Ren, Y. G., Zhang, Z. W., Yu, S. W., and Zhang, Y. G. (2018) Oncogen ic Activation of Nrf2, Though as a Master Antioxidant Transcription Factor, Liberated by Specific Knockout of the Full-Length Nrf1 alpha that Acts as a Dominant Tumor Repressor. Cancers 10

13. Zhang, Y. G., Lucocq, J. M., and Hayes, J. D. (2009) The Nrf1 CNC/bZIP protein is a nuclear envelope-bound transcription factor that is activated by t-butyl hydroquinone but not by endoplasmic reticulum stressors. Biochemical Journal 418, 293-310

14. Zhu, Y. P., Zheng, Z., Hu, S., Ru, X., Fan, Z., Qiu, L., and Zhang, Y. (2019) Unification of Opposites between Two Antioxidant Transcription Factors Nrf1 and Nrf2 in Mediating Distinct Cellular Responses to the Endoplasmic Reticulum Stressor Tunicamycin. Antioxidants 9

15. Snaebjornsson, M. T., Janaki-Raman, S., and Schulze, A. (2020) Greasing the Wheels of the Cancer Machine: The Role of Lipid Metabolism in Cancer. Cell Metab 31, 62-76

16. Kronen berg, F., Schoenborn, V., Heid, I. M., Vollmert, C., Lingenhel, A., Adams, T. D., Hopkins, P. N., Illig, T., Zimmermann, R., Zechner, R., and Hunt, S. C. (2005) The ATGL gene is associated with free fatty acids, trigylcerides and type 2 diabetes. Genet Epidemiol 29, 259-259

17. Memon, R. A., Tecott, L. H., Nonogaki, K., Beigneux, A., Moser, A. H., Grunfeld, C., and Feingold, K. R. (2000) Up-regulation of peroxisome proliferator-activated receptors (PPAR-alpha) and PPAR-gamma messenger ribonucleic acid expression in the liver in murine obesity: Troglitazone induces expression of PPAR-gamma-responsive adipose tissue-specific genes in the liver of obese diabetic mice. Endocrinology 141, 4021-4031

18. Miura, S., Kai, Y., Kamei, Y., and Ezaki, O. (2008) Isoform-specific increases in murine skeletal muscle peroxisome proliferator-activated receptor-gamma coactivator-1 alpha (PGC-1 alpha) mRNA in response to beta 2-adrenergic receptor activation and exercise. Endocrinology 149, 4527-4533

19. Gao, N., Zhang, Z., Jiang, B. H., and Shi, X. L. (2003) Role of PI3K/AKT/mTOR signaling in the cell cycle progression of human prostate cancer. Biochem Bioph Res Co 310, 1124-1132

20. Wang, C., Hu, L., Zhao, L., Yang, P., Moorhead, J. F., Varghese, Z., Chen, Y., and Ruan, X. Z. (2014) Inflammatory stress increases hepatic CD36 translational efficiency via activation of the mTOR signalling pathway. Plos One 9, e103071

21. Cao, D. Y., Luo, J., Chen, D. K., Xu, H. F., Shi, H. P., Jing, X. Q., and Zang, W. J. (2016) CD36 regulates lipopolysaccharide-induced signaling pathways and mediates the internalization of Escherichia coli in cooperation with TLR4 in goat mammary gland epithelial cells. Sci Rep-Uk 6

22. Berger, J., and Moller, D. E. (2002) The mechanisms of action of PPARs. Annu Rev Med 53, 409-435

23. Zhu, Y. P., Zheng, Z., Xiang, Y., and Zhang, Y. (2020) Glucose Starvation-Induced Rapid Death of Nrf1alpha-Deficient, but Not Nrf2-Deficient, Hepatoma Cells Results from Its Fatal Defects in the Redox Metabolism Reprogramming. Oxid Med Cell Longev 2020, 4959821 
Tumor-Repressing Effect onto the Wnt/beta-Catenin-Dependent and Wnt/beta-Catenin-Independent Signaling Networks in the Human Liver Cancer. Oxid Med Cell Longev 2020, 5138539

25. Zhang, S., Deng, Y., Xiang, Y., Hu, S., Qiu, L., and Zhang, Y. (2020) Synergism and Antagonism of Two Distinct, but Confused, Nrf1 Factors in Integral Regulation of the Nuclear-to-Mitochondrial Respiratory and Antioxidant Transcription Networks. Oxid Med Cell Longev 2020, 5097109

26. Wufur, R., Fan, Z., Liu, K., and Zhang, Y. (2021) Differential yet integral contributions of Nrf1 and Nrf2 in the human antioxidant cytoprotective response against tert-butylhydroquinone as a pro-oxidative stressor. bioRxiv 2021.06.05.447190, doi: https://doi.org/10.1101/2021.1106.1105.447190

27. Widenmaier, S. B., Snyder, N. A., Nguyen, T. B., Arduini, A., Lee, G. Y., Arruda, A. P., Saksi, J., Bartelt, A., and Hotamisligil, G. S. (2017) NRF1 Is an ER Membrane Sensor that Is Central to Cholesterol Homeostasis. Cell 171, 1094-1109 e1015

28. Newman, J. R., and Keating, A. E. (2003) Comprehensive identification of human bZIP interactions with coiled-coil arrays. Science 300, 2097-2101

29. Hou, Y., Liu, Z., Zuo, Z., Gao, T., Fu, J., Wang, H., Xu, Y., Liu, D., Yamamoto, M., Zhu, B., Zhang, Y., Andersen, M. E., Zhang, Q., and $\mathrm{Pi}$, J. (2018) Adipocyte-specific deficiency of $\mathrm{Nfe} 2 \mathrm{l} 1$ disrupts plasticity of white adipose tissues and metabolic homeostasis in mice. Biochem Biophys Res Commun

\section{Figure 1}
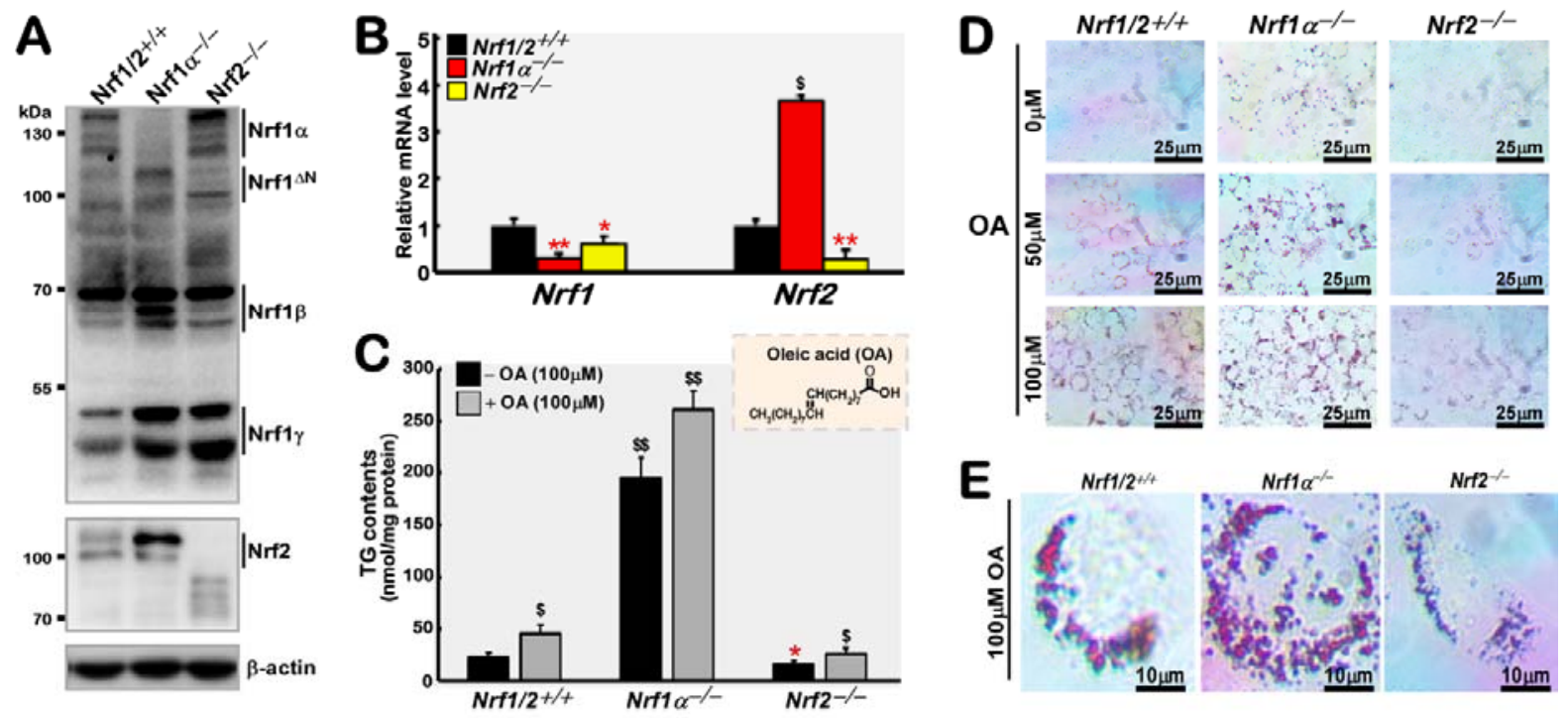

Figure 1. Significant discrepancies between Nrf1 and Nrf2 in lipid metabolism are observed in their genotypic knockout cell lines. (A) Distinct protein abundances of Nrf1 and Nrf2 in three different genotypic cell lines $\mathrm{Nrf1} / 2^{+/+}, \mathrm{Nrf1} \alpha^{-/-}$and $\mathrm{Nrf2}^{-/-}$were determined by Western blotting with their specific antibodies, respectively. (B) Distinct mRNA expression levels of Nrf1 and Nrf2 were examined by real-time qPCR analysis of the aforementioned cell lines. The results are calculated as fold changes (mean \pm S.D) and shown graphically with significant increases $(\$, P<0.05)$ and significant decreases $(*, P<0.05 ; * *, P<0.01)$. (C) The triglyceride (TG) contents were measured after those experimental cell lines were pretreated with $100 \mu \mathrm{mol} / \mathrm{L}$ oleic acid (OA). The results we re calculated as a concentration of $\mathrm{nmol} / \mathrm{mg} \mathrm{protein}$, with significant increases $(\$, P<0.01 ; \$ \$, P<0.001)$ or significant decreases $(*, P<0.01)$. (D) These cell lines were subjected to pretreatment with distinct concentrations of oleic acid $(O A)$, followed by the oil red $O$ staining. The resulting images (scale bar $=25 \mu \mathrm{m}$ ) were then photographed under a microscope. (E) Appropriate sizes of those individual cells that had been treated as described above, were screened by microscopy and photographed. Scale bar $=10 \mu \mathrm{m}$. 


\section{Figure 2}
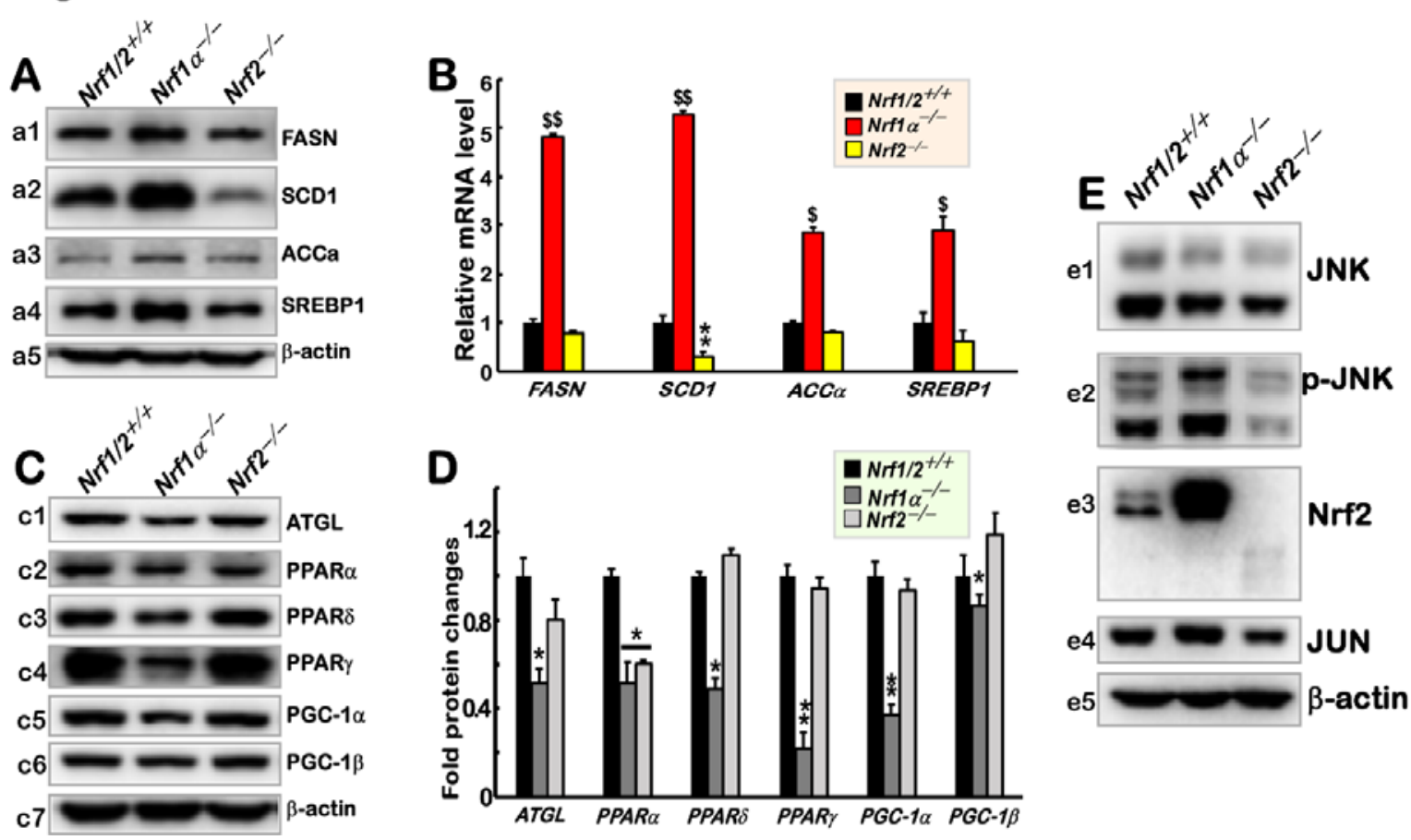

Figure 2. Different expression levels of lipid metabolism-related genes mediated by Nrf1 and Nrf2 in distinct genotypic

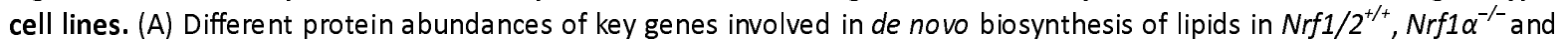
$\mathrm{Nrf2} 2^{-/}$cell lines were determined by Western blotting. (B) Different mRNA expression levels of the indicated genes were also determined by real-time qPCR analysis of the aforementioned cell lines. The results are calculated as fold changes (mean \pm S.D) with significant increases $(\$, P<0.05 ; \$ \$, P<0.01)$ and significant decreases $(* *, P<0.01)$. (C,D) Distinct expression levels of key enzymes and transcription factors involved in lipid catabolism were evaluated by Western blotting (C). The intensity of the indicated immunoblots was calculated at fold changes (mean \pm S.D) and shown graphically with significant decreases $\left(*, P<0.05 ;^{* *}, P<0.01\right)$. (E) Basal protein expression levels of JNK, $p$-JNK, Nrf2 and JUN were also examined in $\mathrm{Nrf1} / 2^{+/+}, \mathrm{Nrf1} \alpha^{-/-}$and $\mathrm{Nrf2}^{-/-}$cell lines. 


\section{Figure 3}
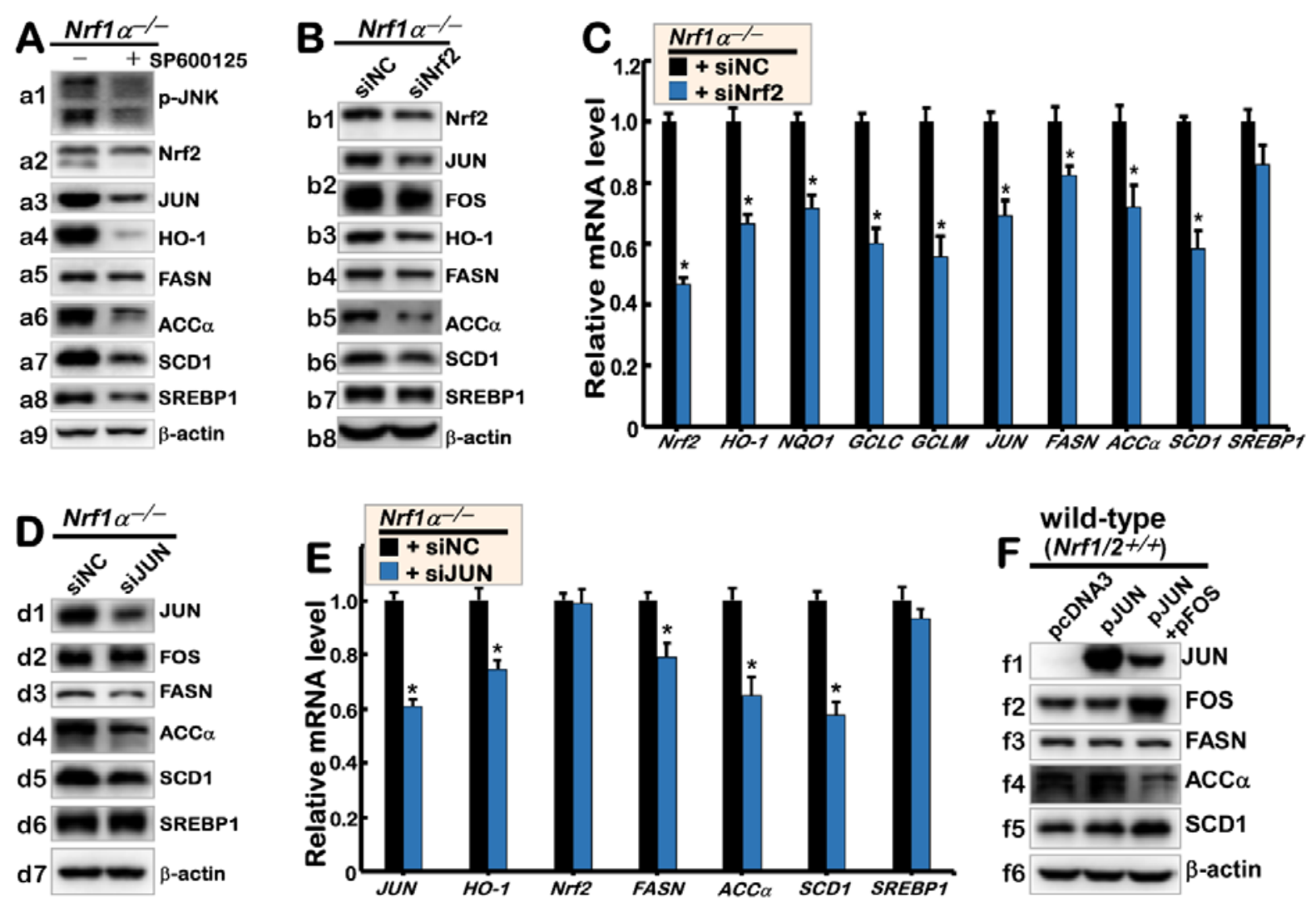

Figure 3. The JNK-Nrf2-AP1 signaling pathway is required for increased expression of lipid synthesis genes in $\mathrm{Nrf1}^{-\alpha^{-}-}$ cells. (A) $\mathrm{Nrf1}^{-/-}$cells were or were not treated with the JNK-specific inhibitor SP600125 (20 $\left.\mu \mathrm{mol} / \mathrm{L}\right)$ for $24 \mathrm{~h}$ and then subjected to Western blotting of the JNK-Nrf2-AP1 signaling towards lipid biosynthesis pathway. (B, C) Nrf1 $\alpha^{-1-}$ cells were transfected with siNC (i.e., small-interfering RNA negative control) or siNrf2 (to specifically interfere with Nrf2), before being determined by (B)Western blotting of the protein abundances as described above or (C) real-time GPCR analysis of their mRNA expression levels. The results were calculated as fold changes (mean \pm S.D) with significant decreases $(*, P<$ 0.01). (D, E) Nrf1 $\alpha^{-/}$cells were transfected with siJUN (to specifically interfere with JUN) or siNC, and subjected to Western blotting analysis (D) and real-time GPCR analysis (E), as described above. (F) Wild-type Nrf1/2 ${ }^{+/+}$cells were allowed for transient transfection with JUN ( $2 \mu \mathrm{g}$ of DNA) alone or plus FOS (i.e., JUN+FOS, every $1 \mu \mathrm{g}$ of DNA) and then subjected to visualization by Western blotting analysis of those indicated proteins. 


\section{Figure 4}

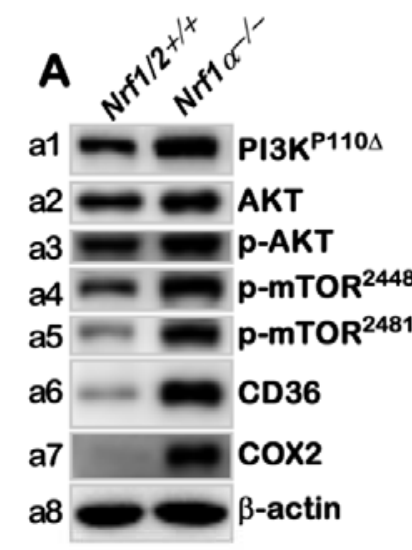

D

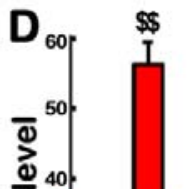

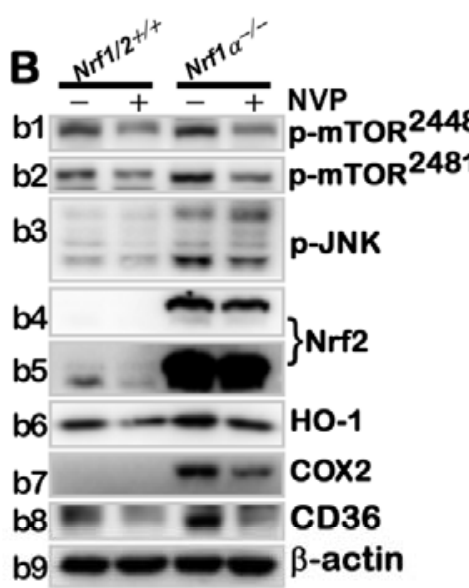

$\mathrm{Nrf1/2+1+}$ Nrf1 $\alpha-1-$

Figure 4. $\mathrm{Nrf1}^{-/-}$cell-autonomous up-regulation of CD36 and COX2 through the PI3K-AKT-mTOR signaling pathway. (A) Western blotting analysis of those indicated protein abundances in both $\mathrm{Nrf1} / 2^{+/+}$and $\mathrm{Nrf1} \alpha^{-/-}$cell lines was carried out as described in the legend for Figure 3. (B, C) These two cell lines were treated with (B) $0.5 \mu \mathrm{mol} / \mathrm{L}$ of the PI3K-specific inhibitor NVP-BKM120 or (C) $200 \mathrm{nmol} / \mathrm{L}$ of the mTOR inhibitor rapamycin (RAPA) for $24 \mathrm{~h}$ and then determined by Western blotting with those indicated antibodies. (D) The mRNA expression levels of key inflammatory factors and cytokines in $\mathrm{Nrf1} / 2^{+/+}$and $\mathrm{Nrf1} \alpha^{-/-}$cell lines were measured by real-time qPCR analysis. The results were calculated as fold changes (mean \pm S.D) with significant increases $(\$, P<0.01 ; \$ \$, P<0.001)$. (E) Western blotting of some inflammatory factors and cytokines revealed changed levels of their protein expression in $\mathrm{Nrf1} / 2^{+/+}$and $\mathrm{Nrf1} \alpha^{-/-}$cell lines. 


\section{Figure 5}
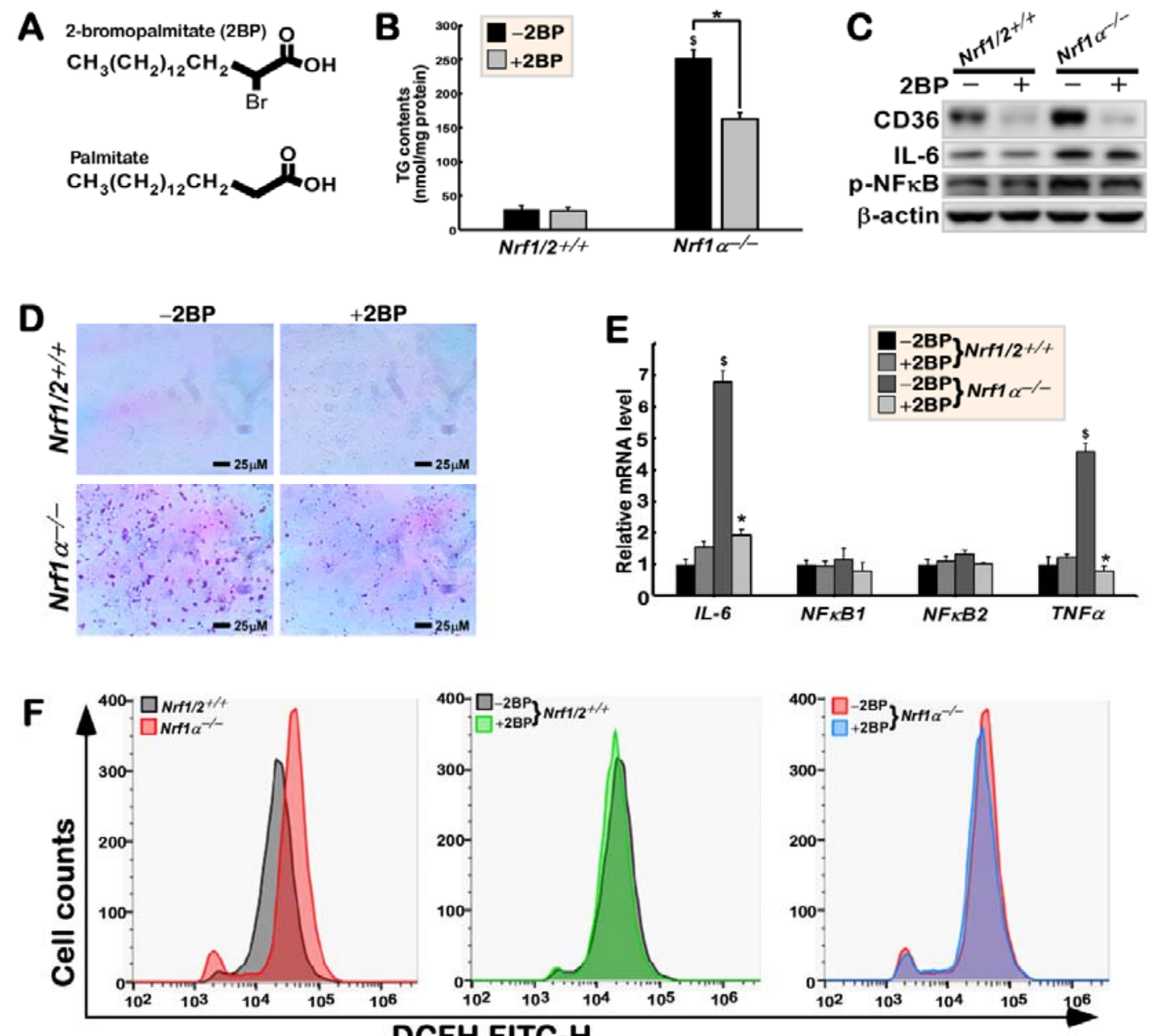

Figure 5. Accumulation of lipids and ROS along with the inflammatory response in $\mathrm{Nrf1}^{-/-}$cells are substantially alleviated by 2-bromopalmitate. (A) The schematic shows two molecular structures of palmitic acid (PA) and 2-bromopalmitate (2BP). (B) The triglyceride contents were measured following treatment of both $\mathrm{Nrf1} / \mathrm{2}^{+/+}$and $\mathrm{Nrf1}^{-/-}$cell lines with $50 \mu \mathrm{mol} / \mathrm{L}$ 2BP. Thereafter, such 2BP-treated cell lines were subjected, respectively, to (C) Western blotting analysis of CD36, IL-6 and p-NFKB; (D) visualization by the Oil red $O$ staining as shown in the photographed images (scale bar $=10 \mu \mathrm{m}$ ); $(\mathrm{E})$ real-time qPCR analysis of IL-6, NFKB1, NFKB2 and TNF $\alpha$ at their $\mathrm{mRNA}$ expression levels, the resulting data of which were calculated as fold changes (mean \pm S.D) with a significant increase $(\$, P<0.01$, relative to the wild-type controls), and another significant decrease $(*, P<0.01$, when compared to those measured from the untreated cells); and (F) flow cytometry determination of ROS as described in the section of "Materials and Methods". 


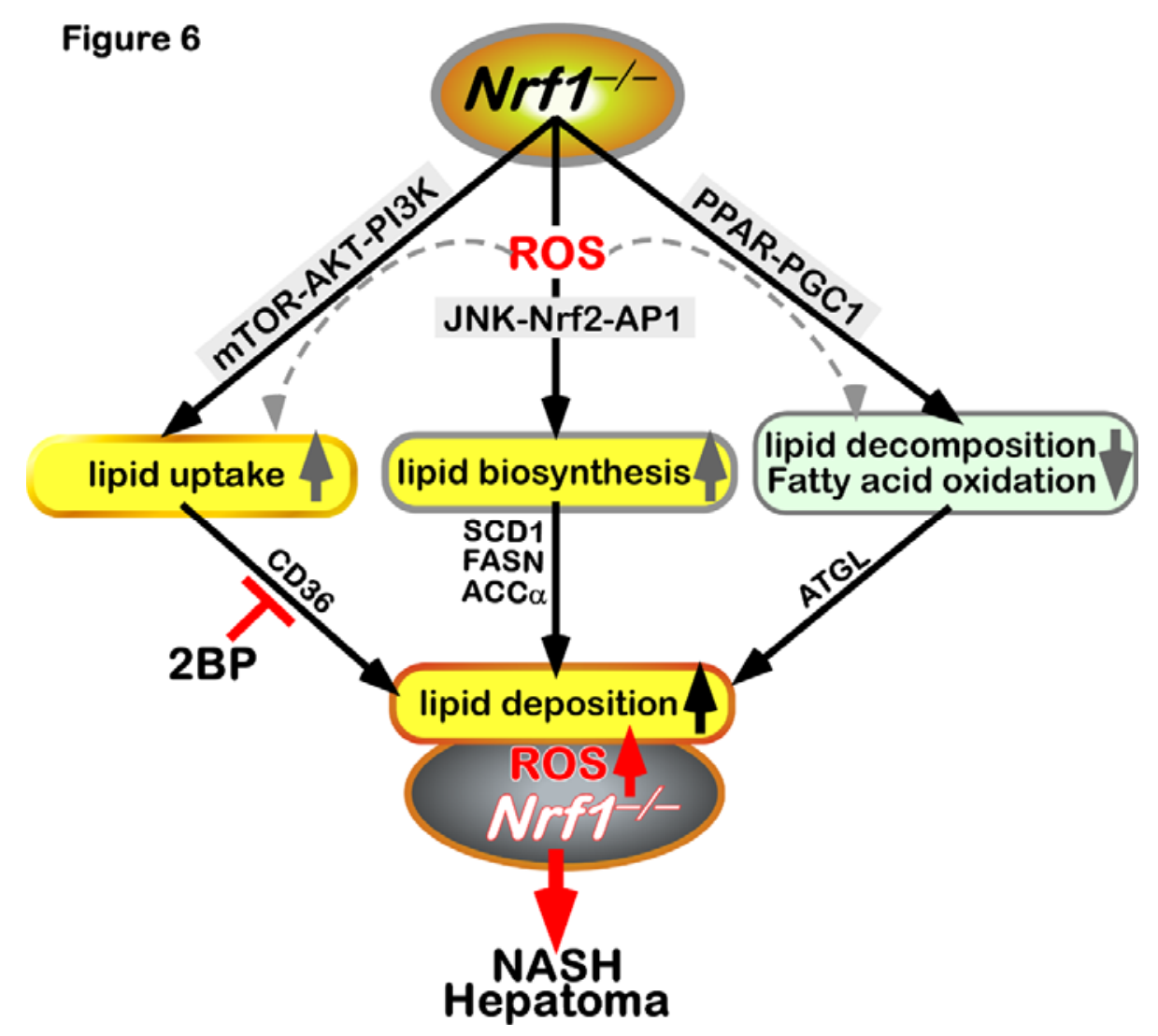

Figure 6. A model proposed for the molecular mechanisms underlying the inflammatory accumulation of lipids and ROS in $\mathrm{Nrfl}^{-/-}$cells. This cartoon provides a better explanation of molecular mechanisms by which the inflammatory accumulation of lipids and ROS occurs in $\mathrm{Nrf1}^{-/-}$cells. Of note, $\mathrm{Nrfl}^{-/ /}$cell-autonomous induction of the PI3K-AKT-mTOR signaling to up-regulation of CD36 results in augmented uptake of fatty acids. The de novo biosynthesis of lipids is up-regulated by the JNK-Nrf2-AP1 signaling to transactivation of their target genes encoding key metabolic rate-limiting enzymes and the relevant transcription factors, while the lipid oxidative decomposition pathway is down-regulated by the

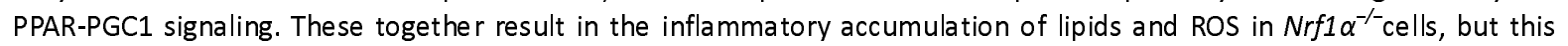
pathological process is alleviated substantially by 2-bromopalmitate (2BP) to down-regulate the CD36 expression. In addition, the yield of ROS in $\mathrm{Nrfl}^{-/-}$cells, was discussed in the relevant main text. 